\title{
Metáforas para navegar en la web ${ }^{\star}$
}

\author{
Guillermo León Zapata Montoya*
}

Recibido: 20 de febrero de 2012 Aprobado: 27 de marzo de 2012

\section{Resumen}

Con la consolidación de Internet y la comunicación permanente que ha generado a través de las redes sociales, los mismos internautas han creado un lenguaje propio que, en muchos de los casos, se ha traducido en una manera connotativa de llamar las cosas. La tesis doctoral "Posibilidades del aprendizaje autónomo a partir del estudio cibermultimedial: caso universitarios de Medellín", ha evidenciado el uso recurrente de metáforas durante las conversaciones de quienes navegan en la WEB, para denominar la acción, procedimiento o actividad que llevan a cabo en el mundo virtual. Este tema es el que se desarrolla en el artículo, como un avance parcial del trabajo investigativo referenciado.

Palabras clave: Internet, metáforas, web 2.0, imaginario, virtualidad.

* El presente artículo hace parte de la investigación denominada "Posibilidades del aprendizaje autónomo a partir del estudio cibermultimedial: caso universitarios de Medellín". El trabajo se hace para la Red de Universidades Públicas, RUDECOLOMBIA y la Universidad Tecnológica de Pereira, en donde realizo el Doctorado en Ciencias de la Educación, línea pensamiento educativo y comunicación.

** Candidato a Doctor en Ciencias de la Educación, Línea Pensamiento Educativo y Comunicación de la Universidad Tecnológica de Pereira, Colombia. Magíster en Educación, de la Universidad de Manizales, Colombia. Especialista en Gerencia de la Comunicación de la Universidad Pontificia Bolivariana, Medellín-Colombia. Comunicador Social-Periodista de Universidad de Antioquia, MedellínColombia.Actualmente se desempeña como Decano de la Facultad de Comunicación Audiovisual del Politécnico Colombiano Jaime Isaza Cadavid, director del grupo de investigación GESTAS e integrante del grupo de investigación COMAEFI. Autor del Libro "Comunicación estratégica para organizaciones". Coautor de la publicación "Caja de herramientas para la didáctica y la pedagogía". Correo electrónico: glzapata@elpoli.edu.co 


\title{
Metaphors for Navigating on the Web
}

\begin{abstract}
As Internet and the permanent communication it has generated through social networks consolidate, same navigators have created a specific language which, in many cases, has resulted in a connotative way of naming things. Doctoral thesis "Possibilities of Autonomous Learning from a Cyber-Multimedia Study: University Cases in Medellin," has demonstrated the recurrent use of metaphors during chats among those navigating on the Web intended to denote the action, procedure or activity conducted on the virtual world. This topic has been developed in this article as a partial advance of the already named research work.
\end{abstract}

Key words: Internet; metaphors; web 2.0; imaginary; virtuality. 


\section{Introducción}

La inserción de las nuevas tecnologías de la información y la comunicación en la vida cotidiana de los estudiantes universitarios le ha dado origen a un nuevo imaginario relacionado con la conexión entre ellos e Internet, lo que se ha traducido también en la creación de metáforas que tienen que ver con la denominación de lo que ellos realizan rutinariamente cuando protagonizan actividades de interacción con sus compañeros a través de la WEB 2.0.

El presente artículo, precisamente, da cuenta de lo que está pasando en el mundo de las redes sociales pero, ante todo en la relación que tejen los estudiantes, docentes y en general quienes utilizan el computador y "navegan" en él permanentemente. Las reflexiones que se presentan aquí son un avance de algunas de las conclusiones que preliminarmente ha arrojado la tesis doctoral denominada "Posibilidades del aprendizaje autónomo a partir del estudio cibermultimedial: caso universitarios de Medellín", la misma que trata de observar y analizar la relación de estudio y aprendizaje que en medio de su supuesta soledad teje el estudiante, a través de un equipo de cómputo conectado a una red en el desarrollo de una actividad académica autónoma que tiene como propósito ahondar en el estudio o simplemente hacer una tarea.

Precisamente, a la hora de desarrollar su estudio, teniendo como compañero el computador en frecuencia de Internet, nuestro estudiante echa mano de una o varias posibilidades:

- Hace su estudio o tarea utilizando como única alternativa la ayuda cibermultimedial que le ofrece el Internet.

- Simplemente hace uso de una o pocas posibilidades del Internet, tales como Wikipedia, www.tareas.com o Google, por ejemplo.

- Es posible que el estudiante utilice el ciberespacio para hacer sus tareas pero de igual manera se distrae con cualquiera de esas posibilidades multimediales que le ofrece el equipo.

- Por último, el estudiante solo utiliza el WORD, PDF o simplemente el equipo como procesador de palabras para hacer la tarea, sin utilizar ninguna ayuda adicional de Internet; en cambio, sí lo tendrá en cuenta para ver un vídeo en Youtube, escuchar un tema musical en Ares o simplemente para conversar de manera ocasional, en momentos de descanso, con sus amigos que le esperan en el Messenger

La tesis en mención hace referencia a un problema que se manifiesta claramente, cuando los universitarios de Medellín estudian a través de redes sociales y en un contexto cibermultimedial, lo que podría influir directamente en el proceso de aprendizaje autónomo.

Cuando los universitarios estudian en Internet, es posible que aprendan autónomamente, pero también es posible que no lo hagan. Un estudiante conectado a la red es posible que se vuelva sociable o también asociable; probablemente reafirmará su individualidad o sencillamente desarrollará habilidades para trabajar en equipo por las posibilidades que dan las redes sociales. Hay un exceso de información en Internet y esto podría traer consecuencias negativas, pero también positivas en el proceso de aprendizaje de los universitarios. Incluso, tendrá la posibilidad de jugar con el lenguaje y hacer ejercicios metafóricos como los que aquí se expondrán.

El hecho es que en una supuesta soledad, el universitario con su equipo de cómputo en Internet realiza las tareas o estudia, alejado de su familia, del perro y de la rutina de la casa. He ahí, la soledad creativa e innovadora de Turansky en Simone (Película dirigida por Andrew Niccol en el año 2002). Es muy parecida a la de un estudiante que está 
en casa realizando una labor formativa y autónoma que, con toda seguridad, le dará muchas más posibilidades de crear y de inventar. Es que tal como sugiere Lileya Manrique (2004) "nos referimos a la autonomía en el aprendizaje como aquella facultad que le permite al estudiante tomar decisiones que le conduzcan a regular su propio aprendizaje en función a una determinada meta y a un contexto o condiciones específicas de aprendizaje" (p. 15). Y agrega citando a Bornas (1994) que "una persona autónoma es aquella cuyo sistema de autorregulación funciona de modo que le permite satisfacer exitosamente tanto las demandas internas como externas que se le plantean".

La virtualidad tiene su propia lógica y, además, la tecnología cambia la naturaleza humana. De ahí que el entorno virtual en el que el universitario estudia cibermultimedialmente en casa es absolutamente particular, en la medida que cuando hace la tarea o simplemente estudia, se enfrenta a una realidad especial en donde aparecen otros protagonistas tecnológicos, ciberespaciales, multimediales y comunicacionales, que dan origen a una forma de estudiar y de denominar las cosas, hechos y actividades diferentes, concretando la denominada cibercultura en un espacio educativo específico que en este caso he llamado ACCA (Ambiente casero y cibermultimedial de aprendizaje), el mismo que se ha conectado con Internet, precisamente porque los estudiantes de este nuevo milenio lo realizan todo o mucho de ello, pegados de la WEB 2.0 y la multimedia.

En el tema que nos ocupa, se trata del aula que crean los estudiantes cuando el docente orienta una actividad independiente, la misma que organizan en casa, en una cafetería, en el mismo salón de clase, en la oficina o en un sitio que adecua, siempre y cuando tenga conexión a la red, y permita la relación multimediática, nuevo paradigma pedagógico que hace presencia en el proceso de enseñanza-aprendizaje en esta parte del mundo.
Han aparecido las nuevas herramientas cibermultimediales que se incrustan en los procesos de aprendizaje autónomo de los estudiantes universitarios que se sientan frente al computador a realizar la tarea, para lo cual echan mano de múltiples posibilidades tecnológicas a partir del uso de Internet, a las mismas que les asignan nombres y funciones de otros contextos, creando un lenguaje propio del mundo virtual.

Google, wikipedia, Ares, redes sociales electrónicas (Twitter, Facebook), tareas.com, hi5, Youtube, la prensa virtual, la cámara, Skype, el micrófono, el messenger, monografías.com, entre otros, son algunas herramientas de uso rutinario de los estudiantes, quienes en el mismo momento las utilizan para estudiar, recrearse, enamorar, hacer la tarea y descansar, constituyendo esto, una nueva manera de asumir el proceso de formación, por la vía de la autonomía cibermultimedial.

Hasta el momento no existen estudios que se ocupen de interpretar la relación estudiantecibermultimedia, como actividad de aprendizaje autónomo que se realiza en casa, y en donde el educando se enfrenta a un equipo de cómputo conectado a una red, en el cual puede escuchar música, ver videos, leer la prensa, conversar y verse con otra persona que está en un lugar diferente, hacer consultas virtuales y realizar la tarea; todo al mismo tiempo.

Esa es la investigación que plantea una tesis sobre el estudio simultáneo en la WEB y en el computador; en medio de ella han aparecido nuevas reflexiones relacionadas con distintos temas como es el caso de las metáforas, que es precisamente la inquietud que desarrollará este artículo en las líneas siguientes:

\section{Las metáforas desde la virtualidad}

Hay un paquete de metáforas que ya hace parte del léxico común y corriente de las instituciones educativas. Por ejemplo, se "requiere bajar 
la información" para lo cual será necesario "navegar en Internet" y "buscar en Google" unos datos "que no se pueden perder". De todas maneras para realizar la tarea tendrá que "registrarse en la página". Además, esa persona precisa de "un encuentro en Facebook" con su amiga que en ese momento "estará on líne"", con la cual también podrá "Chatear en el Messenger de Hotmail". En ese mismo instante, esa persona "abrirá su correo" para "bajar y leer los mensajes" que le han enviado. Es claro que una y otra protagonistas del acto comunicativo-pedagógico están en "un ambiente virtual".

Pero ese nuevo imaginario, tiene muchas más expresiones originadas en la rutina del ordenador, tales como "conéctate en la noche para que hablemos de la tarea..."; claro que, antes de ello, esa persona pide el favor de "linkeame el documento de matemáticas". En otro contexto de la misma lógica virtual, se pide "rotar la foto por el Facebook y el correo" o "etiquetar un video".

Sin duda alguna, estas metáforas pertenecen a una nueva lógica, que tal como lo plantea Emmanuel Lizcano (2006, p.12), es "... la del imaginario. Esa forma, que atenta contra todos los principios lógicos, no es, evidentemente, accesible de modo de directo. Pero sí puede entreverse a través, precisamente, de la manera en que unas metáforas enlazan con otras, la forma como unas llevan a otras, o bloquean la aparición de otras, la manera en que unas entran en conflicto con otras...".

Por ello, conviene en este artìculo tratar de "ganar claridad" sobre lo que significa lo imaginario, en tanto las metáforas de la virtualidad que aquí se plantean le apuntan a una lógica que se origina desde el mismo concepto, porque tal como lo escribió Lizcano (2006), "[...] el concepto de 'imaginario' aún está instituyéndose, y está por tanto en nuestra mano el irle dotando de unos u otros contenidos. [...]". Y es claro, "[...] lo imaginario no es susceptible de definición, por la sencilla razón de que es él la fuente de las definiciones. [...]" Lo imaginario excede cuanto de él pueda decirse pues es a partir de él que puede decirse lo que se dice. [...]" (Lezcano, 2006, p.23). Digamos entonces que si el imaginario es el lugar de la creatividad social, no lo es menos de los límites y fronteras dentro de los cuales cada colectividad, en cada momento, puede desplegar su imaginación, su reflexión y sus prácticas. Este es el tema que se tratará de desarrollar en las líneas siguientes.

Para empezar, será necesario rescatar los planteamientos del autor de las "Metáforas que nos interpretan", Emmanuel Lizcano, quien escribió que "[...] la investigación de las metáforas comunes a una colectividad [es] un modo privilegiado de acceder al conocimiento de su constitución imaginaria." Por lo cual, agrega que "[...] En lo imaginario echan sus raíces dos tensiones opuestas, si no contradictorias. Por un lado, [...] el deseo de utopía. Por otro, el conjunto de creencias consolidadas, de prejuicios, de significados instituidos, de tradiciones y hábitos comunes, sin los que no es posible forma alguna de vida común" (p. 33).

Es bajo este marco teórico y estas reflexiones que se pretende interpretar ese nuevo mundo de la palabra y la cultura que surge con las NTIC y especialmente con la tecnología digital, lo que se ha traducido en un imaginario específico de lo virtual que se presenta metafóricamente en el lenguaje cotidiano de los estudiantes universitarios. Es que, tal como lo planteara Nietzsche y desarrollara Derrida, bajo cada concepto, imagen o idea late una metáfora, una metáfora que se ha olvidado que lo es. Claro que sí; la metáfora es esa tensión entre dos significados, ese percibir el uno como si fuera el otro pero sin acabar de serlo.

Precisamente sobre este tema, Lakoff y Johnson (1980) han escrito que nuestros conceptos "se estructuran metafóricamente, la actividad se estructura metafóricamente y, en consecuencia, el 
lenguaje se estructura metafóricamente" (p. 43), es decir, que la metáfora no está meramente en las palabras que usamos, está en nuestros mismos conceptos porque en definitiva: concebimos las cosas de manera metafórica.

Lo planteado en las líneas anteriores no es ajeno a la realidad que se vive hoy en el mundo universitario en una cafetería en donde confluyen decenas de estudiantes quienes comparten (con o sin su portátil), comentarios, reflexiones y tareas relacionados con la academia, para lo cual "echan mano" de un vocabulario absolutamente novedoso, bastante joven (no mayor a 20 años) y nacido de la práctica misma en la WEB, la virtualidad y la tecnología, las que han dado origen a un imaginario cibercultural, en donde los ahora protagonistas integran con facilidad el mundo físico con el virtual. Veamos:

Los estudiantes universitarios dicen que "se requiere bajar la información". Esta es una metáfora en donde se utilizan dos expresiones de realidades diferentes: Bajar e información. La primera da cuenta del espacio y la segunda del lenguaje. Las dos se han combinado para referirse fundamentalmente a la consecución de datos originados en la WEB.

De igual manera, un universitario le plantea a otro, "vía internet": "Conéctate en la noche para que hablemos de la tarea..."; Claro que, antes de ello, esa persona pide un favor en el cual figura la expresión: "linkeame el documento de matemáticas". En la primera se utiliza la expresión "Conéctate" relacionada normalmente con electricidad aunque aquí se plantea desde la perspectiva de abrir un correo que, entre otras cosas, no es fisico. Es decir, que aparecen dos realidades diferentes las cuales, normalmente, no tendrían que estar relacionadas. En todo caso, lo que significa es que esas dos personas se van a encontrar a determinada hora, pero no de una manera física: lo harán a través del Messenger o Facebook, lo que sugiere otra metáfora.
Y cuando se hace relación a "Linkear el documento", también aparecen dos situaciones de campos diferentes: el LINK, que sí es una expresión relacionada con el espacio virtual, y el documento, que sí tiene que ver con el libro, es decir, con un material escrito. El nuevo imaginario de la virtualidad las integra para expresar que una persona le va a enviar una dirección electrónica a otra por Internet. Muy diferente a la interpretación real.

En otro contexto de la misma lógica virtual, se pide "rotar la foto por el Facebook y el correo" o "etiquetar un vídeo". Lo primero plantea la rotación, que en todo caso es física, pero acá se sugiere hacerlo por el Facebook, es decir, a través de una red virtual. Lo que se sugiere es hacer conocer la foto de varias personas a través de Internet. Y cuando se "etiqueta un vídeo", no es que se esté pasando por el puesto de venta para marcarlo, sino que se está asignando el nombre para leerlo.

Pero bueno. Si se quiere "Bajar la información", lo que se está sugiriendo no es enviar el periódico al piso, sino obtener un documento de los millones que aparecen en Google o en cualquier otro buscador.

Unos datos "que no se pueden perder", hace referencia a un documento que está en un archivo específico virtual o real en el computador y que si no se tiene cuidado, se borra.

Y cuando hay un "encuentro en Facebook", no es que haya un cita en la sede de la empresa multinacional de servicios informáticos de redes sociales en Estados Unidos, sino que varias personas "abrirán el sitio" (es decir, pondrán en funcionamiento su Internet) y conversarán por esa vía a determinadas horas.

\section{Conclusión}

En fin, son miles y miles las metáforas que pertenecen a este nuevo imaginario de lo virtual, y las mismas que ya deambulan por los léxicos 
específicos de quienes a diario usan la WEB 2.0 para conversar.

De todas formas, hay quienes plantean como Alan Paulos (2004), que "la metáfora ha de entenderse, creo yo, como algo que no aporta nada sustancialmente nuevo, pues la información trasmitida se halla demasiado condicionada a lo que ya se sabe en terrenos ajenos a lo que se quiere explicar (por ello, las metáforas ni siquiera son ni pueden ser redundantes, respecto a lo que quieren explicar, sin dejar de ser metáforas propiamente dichas)" (p. 18).

Ahora bien, en el caso concreto de los temas tecnológicos y específicamente sobre Internet y los computadores, me quiero alejar de otros planteamientos del mismo Alan Paulos (2004), quien escribió que "siempre que un pasaje tiene un componente metafórico (...), o cuando se depende de un contexto o un conocimiento de fondo, surgen problemas con los ordenadores" (p. 23). Es decir, el aparato en sí no podría funcionar con metáforas, como en ciertos casos científicos, pero la conexión a la red, y sobre todo el lenguaje y diálogo entre los protagonistas de la relación sí se da cotidianamente a través de esas "maromas" que construyen los hablantes para dar cuenta de su actividad rutinaria en la red. Quizá, la aparente necesidad del ser humano de construirse universos simbólicos y su facilidad para cambiar su contenido sin variar su estructura tenga mucho que ver con ese aprendizaje metafórico.

De acuerdo con todo lo expuesto, y teniendo en cuenta los planteamientos de de Lizcano (2006),
Para que una metáfora nueva, o una constelación de metáforas, exprese -0 impulse- un cambio en el imaginario son necesarias al menos tres condiciones. Primero, es necesario que esa metáfora sea imaginable o verosímil desde un imaginario dado, [...] Segundo, hace falta también que la metáfora viva, una vez concebida, encuentre un caldo de cultivo adecuado para crecer y consolidarse [...] Una forma habitual de generar metáforas vivas que, no obstante, obtengan cierto consenso social es alterar o invertir una determinada constelación de metáforas zombis. Por ejemplo, pueden invertirse todas las metáforas que, en el imaginario ilustrado, localizaban el tiempo y generar así un imaginario anti-ilustrado. En lugar de "atados al pasado" podemos hablar de estar "atados al futuro" y, de repente, toda una serie de figuras irrumpen en el escenario [...]. En tercer y último lugar, no es menos necesario que esa metáfora desbanque a otras [...] La lucha por el poder es, en buena medida, una lucha por imponer las propias metáforas (p. 48).

Lo anterior indica que el imaginario del lenguaje virtual y las conexiones de Internet existen, en la medida que los estudiantes universitarios utilizan las metáforas desde la especificidad de la relación pedagógica, del uso del lenguaje y del encuentro entre jóvenes; tiene también un espacio en donde consolidarse, que es precisamente el de la WEB; por último, es claro que estas metáforas virtuales han desplazado la interacción y comu nicación oral y presencial que cotidianamente tejen los estudiantes para estudiar y/o realizar la tarea. 


\section{Referencias bibliográficas}

Ceballos, V. (2010). La investigación en educación. Maracaibo.

Herrera, P. (2011). Notas de clase. Seminario de Tesis. Doctorado en Pensamiento Educativo y Comunicación. Pereira: Universidad Tecnológica de Pereira.

Lizcano, Emmanuel (2006). Metáforas que nos piensan. Madrid: Traficantes de sueños. Ediciones Bajo Cero.
Lakoff, G. y Johnson, M. (1980). Metáforas de la vida cotidiana. Chicago: Universidad de Chicago.

Manrique Villavicencio, L. (2004). El aprendizaje autónomo en la educación a distancia. Primer congreso Latinoamericano virtual de educación a distancia. Departamento de Educación, Pontificia Universidad Católica del Perú.

Paulos, A. (2004). Un matemático invierte en Bolsa. La Sorbona. 